\title{
Evolution of the system with multiplicative noise
}

\author{
Alexander I. Olemskoi*, Dmitrii O. Kharchenko \\ Physical Electronics department, Sumy State University, 2, Rimskii-Korsakov St., 40007 Sumy Ukraine
}

Received 24 March 2000; received in revised form 13 November 2000

\begin{abstract}
The governed equations for the order parameter, one- and two-time correlators are obtained for systems with white multiplicative noise. We consider the noise whose amplitude depends on stochastic variable as $x^{a}$ where $0<a<1$. It turns out that the equation for autocorrelator includes an anomalous average of the power-law function with the fractional exponent $2 a$. Determination of this average for the stochastic system with a self-similar phase space is performed. It is shown that at $a>\frac{1}{2}$, when the system is disordered, the correlator behaves in the course of time non-monotonically, whereas the autocorrelator increases monotonically. At $a<\frac{1}{2}$ the phase portrait of the system divides into two domains: at small initial values of the order parameter, the system evolves to a disordered state, as above; within the ordered domain it is attracted to the point with finite values of the autocorrelator and order parameter. The long-time asymptotes are defined to show that, within the disordered domain, the autocorrelator decays hyperbolically and the order parameter behaves as a power-law function with fractional exponent $-2(1-a)$. Correspondingly, within the ordered domain, the behaviour of both dependencies is exponential with an index proportional to $-t \ln t$. (C) 2001 Elsevier Science B.V. All rights reserved.
\end{abstract}

PACS: $05.40 .+\mathrm{j} ; 64.60 .-\mathrm{i}$

Keywords: Stochastic system; Order parameter; Correlator

\section{Introduction}

Within the framework of the usual thermodynamic approach, it is postulated that the bath is passive with respect to a variation of the parameter $x$ which is an amplitude of the hydrodynamic mode [1]. In this case, the noise is additive in nature and its intensity does not depend on the stochastic variable $x$. On the contrary, a multiplicative noise has an amplitude $g(x)$ as a function of the variable $x$. Investigations of such kinds of

\footnotetext{
* Corresponding author.

E-mail addresses: alexander@olem.sumy.ua, olemskoi@ssu.sumy.ua (A.I. Olemskoi), dikh@ssu.sumy.ua (D.O. Kharchenko).
} 
systems were performed for population dynamics [2], directed percolation [3], Lévy flights [4] and so on.

Nowadays, a great interest in statistical physics is connected to systems with selfsimilar phase space. The behaviour of such a system is known to be defined in terms of homogeneous functions [5]. In the simplest case, we can use the power-law function for the noise amplitude [6]:

$$
g(x)=x^{a}, \quad a \in[0,1] .
$$

This kind of assumption allows us to consider a whole set of models. Indeed, for the ordinary thermodynamic system with an additive noise one has $a=0$, for the directed percolation process $a=\frac{1}{2}$, and in the case of population dynamics $a=1$. We will investigate temporal behaviour of the first statistical moments for arbitrary exponent $a$ in stochastic system driven by the deterministic force defined by $x^{4}$-potential.

This paper is organized as follows. In Section 2 the governed equations for the first moment of the stochastic variable $x(t)$ as well as the one- and two-time correlators are obtained on the basis of the Langevin equation with the white multiplicative noise. It turns out that the equation for autocorrelator acquires an anomalous average of the squared power-law function (1) with fractional exponent $2 a$. Section 3 deals with the determination of this average for the stochastic system, whose distribution function is a homogeneous one and can be approximated by a power-law function [6]. In Section 4 we will show that at $a>\frac{1}{2}$ the system is disordered and represented by correlator and autocorrelator only. According to Section 5, at $a<\frac{1}{2}$ we ought to take into account the evolution of the order parameter as well. Finally, Section 6 contains a short conclusion.

\section{Basic equations}

As usual, let us start with the Langevin equation

$$
\frac{\mathrm{d} x}{\mathrm{~d} t}=f(x)+g(x) \xi(t)
$$

for a stochastic variable $x(t)$. On the right-hand side of Eq. (2), $f(x)$ is the deterministic evolution force, the second term defines the multiplicative noise with the amplitude $g(x)$. The statistical properties of the Langevin force $\xi(t)$ are standard:

$$
\langle\xi(t)\rangle=0, \quad\left\langle\xi(t) \xi\left(t^{\prime}\right)\right\rangle=\delta\left(t-t^{\prime}\right),
$$

where the angle brackets denote the averaging. In order to study the simplest properties of the system, we consider the order parameter $\eta(t) \equiv\langle x(t)\rangle$, the two-time correlator $G\left(t, t^{\prime}\right) \equiv\left\langle\delta x(t) \delta x\left(t^{\prime}\right)\right\rangle, \delta x(t) \equiv x(t)-\langle x(t)\rangle$ and the autocorrelator $S(t) \equiv\left\langle(\delta x(t))^{2}\right\rangle$. Within the framework of the white-noise approximation (3) and the Ito calculus, we can treat $g(x)$ and $\xi(t)$ as statistically independent functions. Then, averaging Eq. (2) under the condition

$$
\langle g(x) \xi(t)\rangle=0,
$$


we obtain the evolution equation for the first moment: $\dot{\eta}=\langle f(x)\rangle$ where $\langle f(x)\rangle \neq$ $f(\eta)$, dot stands for the derivative with respect to time $t$. Without loss of generality, the deterministic part of the evolution force can be chosen in the polynomial Landau form

$$
f(x)=-\frac{\partial V(x)}{\partial x}, \quad V(x)=-\frac{\varepsilon}{2} x^{2}+\frac{1}{4} x^{4},
$$

where $\varepsilon$ is a parameter that acts as the dimensionless temperature, counted off a critical value. The averaging of the force (5) gives term $\left\langle x^{3}\right\rangle$ that is reduced to $\eta\left\langle x^{2}\right\rangle \equiv \eta\left(\eta^{2}+\right.$ $S$ ), in accordance with the cumulant expansion. As a result, the evolution equation for order parameter takes the form

$$
\dot{\eta}=\eta\left(\varepsilon-\eta^{2}\right)-3 \eta S,
$$

where the values $\eta, S$ are functions of time $t$.

According to Eqs. (3), (4), we have

$$
\begin{aligned}
\left\langle g(x(t)) x\left(t^{\prime}\right) \xi(t)\right\rangle= & \left\langle g(x(t)) x\left(t^{\prime}\right)\right\rangle\langle\xi(t)\rangle \\
& +\langle g(x(t)) \xi(t)\rangle \eta\left(t^{\prime}\right)+\langle g(x(t))\rangle\left\langle x\left(t^{\prime}\right) \xi(t)\right\rangle=0 .
\end{aligned}
$$

Hence, the equation for two-time correlator takes the form

$$
\frac{\partial}{\partial t} G\left(t, t^{\prime}\right)=\left\{\varepsilon-3\left[\eta^{2}(t)+S(t)\right]\right\} G\left(t, t^{\prime}\right) .
$$

The problem lies now in obtaining an evolution equation for the variance $S \equiv\left\langle x^{2}\right\rangle-\eta^{2}$. Making use of the relation $\mathrm{d} x^{2} \equiv(x+\mathrm{d} x)^{2}-x^{2}$ where the differential $\mathrm{d} x$ is written as

$$
\mathrm{d} x=f(x) \mathrm{d} t+g(x) \mathrm{d} w, \quad \mathrm{~d} w \equiv \xi(t) \mathrm{d} t, \quad(\mathrm{~d} w)^{2}=\mathrm{d} t,
$$

we can write down the equation for $\left\langle x^{2}\right\rangle$ in the following form:

$$
\frac{\mathrm{d}}{\mathrm{d} t}\left\langle x^{2}\right\rangle=2\langle x f(x)\rangle+\left\langle g^{2}(x)\right\rangle .
$$

Here the last term is the average intensity of the multiplicative noise being a result of the interaction of the variable $x$ with a bath, whose variables have been appropriately eliminated. Inserting definition (1) into Eq. (9), we get the average $\left\langle x^{2 a}\right\rangle$ with a fraction exponent $2 a$. Further, we are going to obtain an expression for such a fractional average in terms of the cumulant expansion.

\section{Calculation of the fractional average}

Let us suppose that, apart from the initial distribution $P(x)$, there is another one $P_{q}(x)$ specified by a positive parameter $q<1$. Moreover, for $P(x)$ and $P_{q}(y)$ we assume the following relation:

$$
x^{q} P(x) \mathrm{d} x \equiv y P_{q}(y) \mathrm{d} y .
$$


Then, the normalized distribution $P_{q}(y)$ of the new stochastic variable $y \equiv x^{q}$ takes the form

$$
P_{q}(y)=q^{-1} y^{(1-q) / q} P\left(y^{1 / q}\right) .
$$

If we denote $\langle\cdots\rangle_{q}$ for average over the distribution $P_{q}(y)$ and use the designation $\langle\cdots\rangle$ for average over the initial distribution $P(x)$, then we obtain

$$
\begin{aligned}
\left\langle x^{q}\right\rangle & =\langle y\rangle_{q}, \\
\left\langle x^{q}\right\rangle & \equiv \int x^{q} P(x) \mathrm{d} x, \quad\langle y\rangle_{q} \equiv q^{-1} \int y y^{(1-q) / q} P\left(y^{1 / q}\right) \mathrm{d} y .
\end{aligned}
$$

Thus, making use of the distribution (11), we may take averages of a power-law function $x^{q}, q>1$.

For self-similar stochastic systems the distribution function is a homogeneous function [6] to be written in a power-law form as

$$
P(x) \simeq A x^{-2 a}, \quad A \equiv \frac{1}{2}|1-2 a| b^{|1-2 a|} .
$$

Here $A$ takes care of normalization cut-off procedure

$$
2 \int_{b}^{1 / b} P(x) \mathrm{d} x=1
$$

with $b \rightarrow 0$. Using Eq. (13), we obtain fractional averages of the variable $x$

$$
\begin{aligned}
& \left\langle x^{n q}\right\rangle \equiv A \int x^{n q} x^{-2 a} \mathrm{~d} x=A(1-2 a+n q)^{-1} x^{1-2 a+n q}, \\
& \left\langle x^{n}\right\rangle \equiv A \int x^{n} x^{-2 a} \mathrm{~d} x=A(1-2 a+n)^{-1} x^{1-2 a+n} .
\end{aligned}
$$

Then, Eq. (12) immediately produces

$$
\left\langle x^{n q}\right\rangle=\alpha_{n}(q)\left\langle x^{n}\right\rangle^{p_{n}(q)} .
$$

Here the exponent $p_{n}(q)$ and the multiplier $\alpha_{n}(q)$ are introduced as follows:

$$
p_{n}(q)=\frac{1-2 a+n q}{1-2 a+n}, \quad \alpha_{n}(q)=A^{\frac{n(1-q)}{(1-2 a+n)}} p_{n}^{-1}(q)(1-2 a+n)^{p_{n}(q)-1} .
$$

Now, we are ready to formulate the equation for the autocorrelator $S=\left\langle x^{2}\right\rangle-\eta^{2}$ that will be based on Eqs. (9), (1), (17), (18). According to Ref. [7] a keypoint of the system with the multiplicative noise (1) is that its behaviour is governed by the magnitude of the exponent $a$ in Eq. (1). At $\frac{1}{2}<a<1$, when the fractal dimension of the phase space $D=2(1-a)$ is less than 1 , the system is always disordered and its evolution is represented by the two-time correlator $G\left(t, t^{\prime}\right)$ and the autocorrelator $S(t)$ only. The former is governed by Eq. (7), whereas for the latter we obtain

$$
\begin{aligned}
& \dot{S}=2 S(\varepsilon-3 S)+\alpha_{2} S^{p_{2}}, \\
& \alpha_{2} \equiv \alpha_{2}(a)=A^{2(1-a) p_{2}} p_{2}^{-p_{2}}, \quad p_{2} \equiv p_{2}(a)=(3-2 a)^{-1}
\end{aligned}
$$


from Eqs. (6), (9), (1), (17), (18) with $q=a, n=2$. Within another domain $0<a<\frac{1}{2}$, where the fractal dimension $D>1$, the system can be ordered and instead of Eq. (19) we have

$$
\begin{aligned}
& \dot{S}=2 S\left[\varepsilon-3\left(\eta^{2}+S\right)\right]+\alpha_{1} \eta^{p_{1}}, \\
& \alpha_{1} \equiv \alpha_{1}(2 a)=A^{(1-2 a) p_{1}} p_{1}^{-p_{1}}, \quad p_{1} \equiv p_{1}(a)=[2(1-a)]^{-1}
\end{aligned}
$$

for $q=2 a, n=1$.

\section{Evolution of disordered system}

As pointed out above, for exponent $a>\frac{1}{2}$, when fractal dimension $D<1$, the system is governed by Eqs. (19), (7) for the one- and two-time correlators $S(t), G\left(t, t^{\prime}\right)$ being the autocorrelator and the Green response function. The form of the time dependence for the former is shown in Fig. 1(a). It is seen that $S(t)$ monotonically increases to the stationary magnitude $S_{0}$ determined by the equation

$$
\varepsilon-3 S_{0}+\left(\alpha_{2} / 2\right) S_{0}^{p_{2}-1}=0 .
$$

In the limit $S \ll 1$ when $S^{p_{2}} \gg S \gg S^{2}$, Eq. (19) gives the power-law time dependence

$$
S(t)=\left(\frac{A^{2(1-a)}}{p_{2}\left(1-p_{2}\right)}\right)^{p_{2} /\left(1-p_{2}\right)} t^{1 /\left(1-p_{2}\right)}, \quad p_{2} \equiv(3-2 a)^{-1},
$$

where we put $S(t=0)=0$. In the opposite case $S_{0}-S \ll S_{0}$ one has the exponential dependence $S-S_{0} \propto \mathrm{e}^{-\lambda t}, \lambda \equiv 6\left(2-p_{2}\right) S_{0}-2\left(1-p_{2}\right) \varepsilon$. According to Eq. (21) with the $\varepsilon$ increase the stationary value $S_{0}$ rises from the minimal magnitude $\left(\alpha_{2} / 6\right)^{1 /\left(2-p_{2}\right)}$ (see Fig. 1(b)).

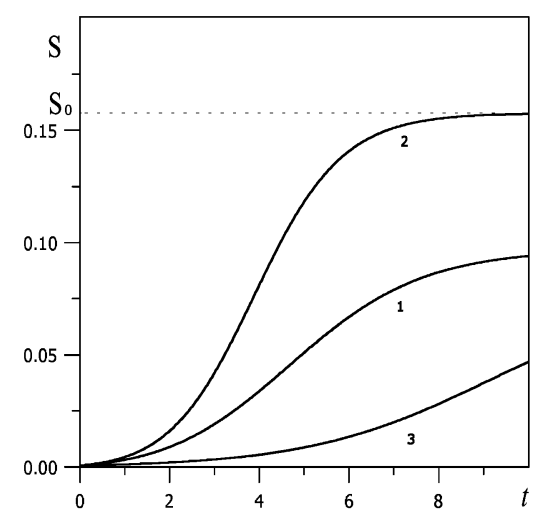

(a)

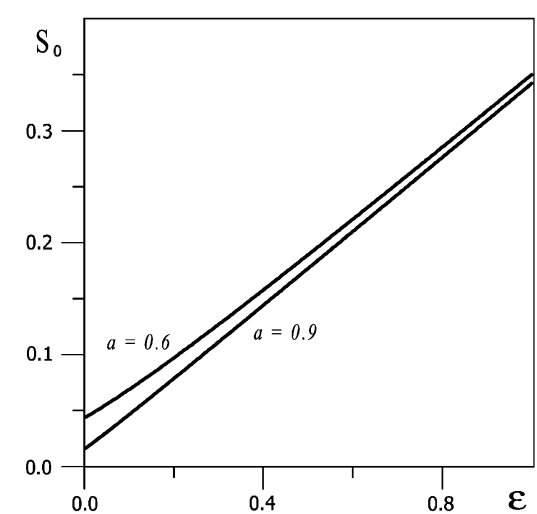

(b)

Fig. 1. Autocorrelator behaviour at $a>\frac{1}{2}$ : (a) time dependencies $S(t)$ (curves $1,2,3$ correspond to $a=0.6, \varepsilon=0.2 ; a=0.6, \varepsilon=0.4 ; a=0.9, \varepsilon=0.2$ ); (b) stationary point $S_{0}$ vs. temperature $\varepsilon$ for several values of exponent $a$. 


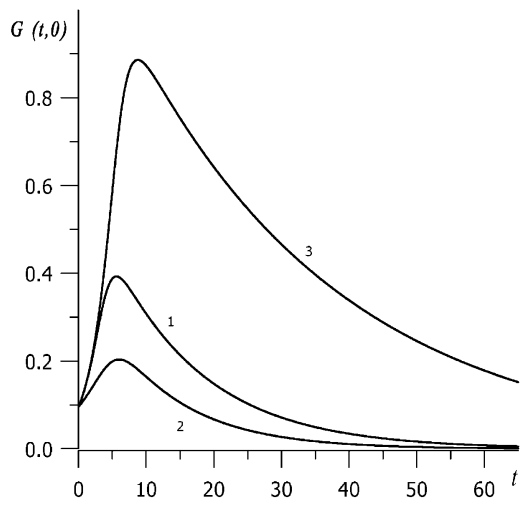

Fig. 2. Correlator $G(t, 0)$ vs. time at $a>\frac{1}{2}$ for several values of exponent $a$ and temperature $\varepsilon$ (curves $1,2,3$ correspond to: $a=0.6, \varepsilon=0.4 ; a=0.6, \varepsilon=0.2 ; a=0.9, \varepsilon=0.4)$.

The solutions of Eq. (7) are shown in Fig. 2 for different values $a$ and $\varepsilon$. We plot correspondent dependencies of the Green function $G(t, 0)$ at identical initial conditions. It is interesting to observe that the correlator $G(t, 0)$ reaches firstly its maximum and then decreases to zero monotonically. For $\varepsilon$ large enough the function $G(t, 0)$ attains its maximum more sharply (cf. curves 1 and 2). The increase in exponent $a$ leads to a similar effect (cf. curves 1 and 3 ).

\section{Evolution of ordering system}

Let us consider now the case $a<\frac{1}{2}$ corresponding to the fractal dimension $D>1$. It provides that the system behaviour is governed by Eqs. (6), (7) and (20), from which the first and third form the closed-loop system of differential equation. To analyse the latter, it is convenient to use the phase plane method. As is seen from the corresponding phase portrait in Fig. 3(a), at small values of $\varepsilon$ there is only one attractive point $\eta_{0}=0$, $S_{0}=\varepsilon / 3$ (Fig. 3(a)). If $\varepsilon$ increases, then the bifurcation creates new saddle and attractive points (see Fig. 4) with coordinates $\eta_{c}=\left[\left(2-p_{1}\right)\left(4-p_{1}\right)^{-1} \varepsilon_{0}\right]^{1 / 2}, S_{c}=(2 / 3)\left(4-p_{1}\right)^{-1} \varepsilon_{0}$ where the bifurcation temperature is given by

$$
\varepsilon_{0}=\frac{4-p_{1}}{2-p_{1}}\left[\frac{3}{8}\left(2-p_{1}\right) \alpha_{1}\right]^{2 /\left(4-p_{1}\right)} .
$$

As can be seen from Fig. 5 the bifurcation temperature $\varepsilon_{0}$ increases infinitely with the exponent $a$ growth to the critical value $a=\frac{1}{2}$. The coordinates of the new stationary points are determined by the following equations:

$$
\varepsilon-\eta_{0}^{2}-\left(\frac{3}{4}\right) \alpha_{1} \eta_{0}^{p_{1}-2}=0, \quad S_{0}=\left(4 \alpha_{1}\right)^{-1}\left(\varepsilon-3 S_{0}\right)^{\left(p_{1} / 2\right)-1} .
$$

To achieve these we put $\dot{\eta}=0, \dot{S}=0$ in Eqs. (6) and (20). Temperature dependencies $\eta_{0}(\varepsilon), S_{0}(\varepsilon)$ of the corresponding steady-state magnitudes are depicted in Fig. 4. Here dashed lines display the saddle point $S$, the solid lines represent the attractive point $C$. 


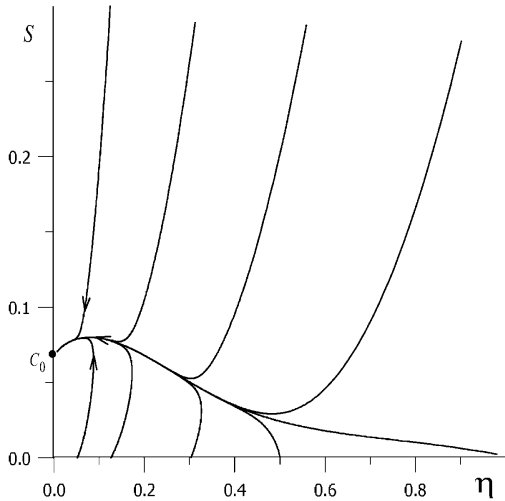

(a)

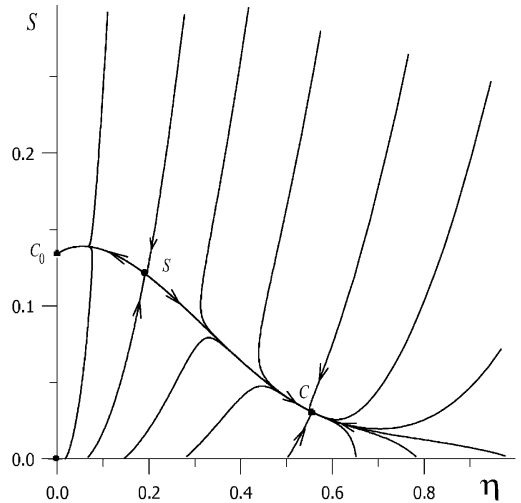

(b)

Fig. 3. Phase portrait at $a>\frac{1}{2}$ : (a) $a=0.3, \varepsilon=0.2$; (b) $a=0.3, \varepsilon=0.4$.

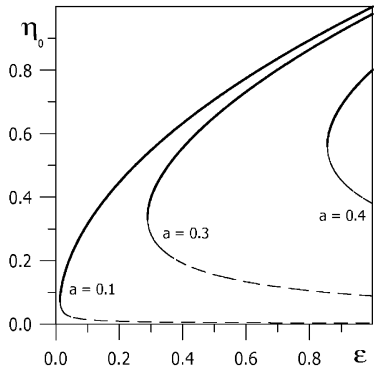

(a)

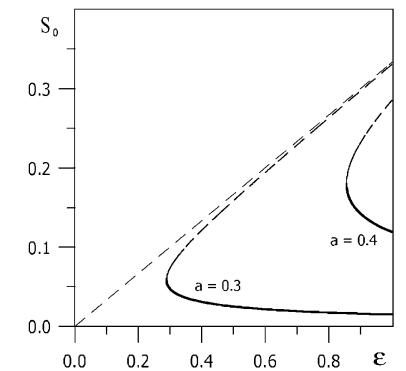

(b)

Fig. 4. Stationary states of the system at $a>\frac{1}{2}$ : (a) order parameter $\eta$ vs. temperature $\varepsilon$ for several values of exponent $a$; (b) autocorrelator $S$ vs. temperature $\varepsilon$ for several values of exponent $a$.

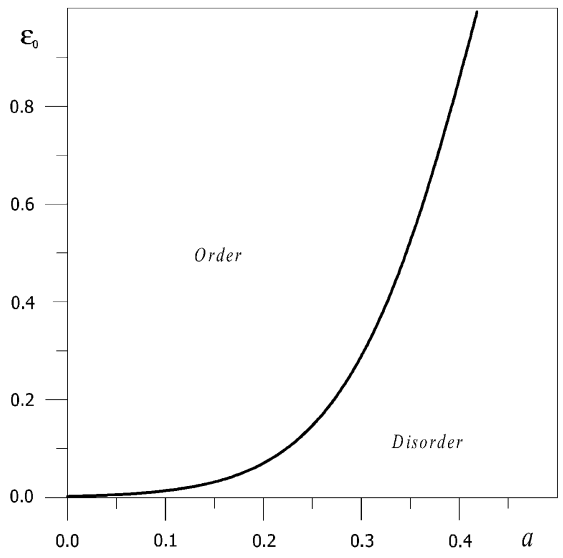

Fig. 5. Phase diagram $\varepsilon_{0}(a)$. 
It is interesting to note that the system undergoes the phase transition of the first order, despite the bare $x^{4}$-potential (5) corresponding to the continuous one. Thus, at small values of the exponent of the multiplicative noise $\left(a<\frac{1}{2}\right)$ the fluctuations transform order of the phase transition, whereas at $a>\frac{1}{2}$ fluctuations suppress the ordering process completely [7].

Now, let us return to analysis of the time dependencies of the main averages under consideration. Firstly, we analyse the system evolution in the disordered state (a vicinity of the point $C_{0}$ in Fig. 3) for extremely large time $t \rightarrow \infty$. The keypoint of our consideration is that the fractional average appearance in Eq. (20) does not allow to use the ordinary Lyapunov method because the exponential time dependence becomes invalid. Instead, let us introduce the generalized Tsallis exponent [8]:

$$
\mathrm{e}^{q t} \rightarrow \exp _{q}(t) \equiv[1+(1-q) t]^{1 / 1-q}
$$

with generalized Lyapunov index $q$. First, such a type of generalization was used by Tsallis [9] to obtain the ordinary Gibbs-Boltzmann exponent in the limit $q \rightarrow 1$. In our case, the latter is arbitrary so that function $\exp _{q}(t)$ acquires the power-law character, in particular the following derivation rule is fulfilled:

$$
\frac{\partial}{\partial t} \exp _{q}(t)=\left(\exp _{q}(t)\right)^{q} \equiv \exp _{q}^{q}(t) \text {. }
$$

In the limits of the short and long times this function has the asymptotic behaviour:

$$
\lim _{t \rightarrow 0} \exp _{q}(t) \rightarrow 1+t, \quad \lim _{t \rightarrow \infty} \exp _{q}(t) \rightarrow[(1-q) t]^{1 / 1-q} .
$$

Below, the first of the asymptotes will be used to extract the Lyapunov-type multipliers, the second one allows us to set an index $q$ (see after Eq. (31)).

Let us assume solutions of Eqs. (6), (20) in the form

$$
\eta(t)=m \exp _{\mu}(t), \quad S(t)=S_{0}+n \exp _{v}(t),
$$

where $S_{0}=\varepsilon / 3$ corresponds to the point $C_{0}$; the exponents $\mu, v$ and coefficients $m, n$ must be determined. ${ }^{1}$ Inserting Eqs. (28) into Eqs. (6), we obtain up to the first power of $m, n \ll 1$ :

$$
3 n \exp _{\mu}^{1-\mu}(t) \exp _{v}(t)=-1 .
$$

From this, within the long-time approximation (27), we obtain

$$
n^{-1}=3(1-\mu), \quad v=2 \text {. }
$$

Similarly, inserting Eq. (28) into Eq. (20) we find

$$
\exp _{v}^{1-v}(t)\left[2 \varepsilon n-\alpha_{1} m^{p_{1}} \exp _{\mu}^{p_{1}}(t) \exp _{v}^{-1}(t)\right]=-n .
$$

As has been pointed out above, in the short-time limit, the function $\exp _{v}^{1-v}(t)$ can be taken equal to 1 to correspond to an extraction of the Lyapunov multiplier. Similarly, in the long-time limit we have $\exp _{\mu}^{p_{1}}(t) \exp _{v}^{-1}(t)=$ const. $\equiv p_{1}^{-1}$ and we obtain

$$
3 \alpha_{1} m^{p_{1}}=-(1+2 \varepsilon), \quad \mu=1+p_{1} \equiv 1+[2(1-a)]^{-1} .
$$

\footnotetext{
${ }^{1}$ It is worthwhile to note that one has $n \ll 1$ because it is the second term in expansion (28), whereas a magnitude $m$ can be arbitrary because it stands as the first term there. Physically, it means that the order parameter behaves in a non-linear manner, in contrast to a linear regime of the autocorrelator.
} 


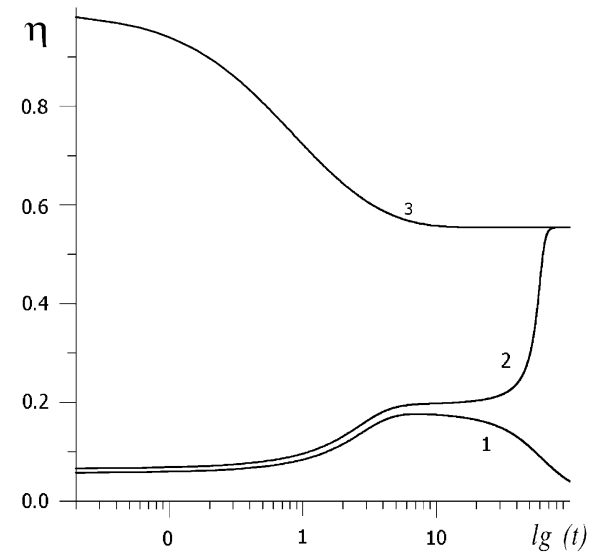

(a)

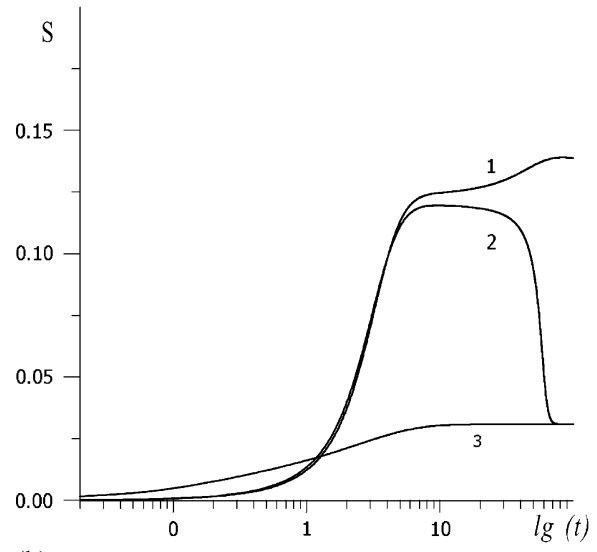

(b)

Fig. 6. Time dependencies corresponding to different trajectories on the phase portrait in Fig. 3: (a) $\eta$ vs. $\lg t$ at $a=0.3, \varepsilon=0.4$ and $S(0)=0$ (curves $1,2,3$ correspond to $\eta(0)=0.057, \eta(0)=0.066, \eta(0)=1.0$ ); (b) $S$ vs. $\lg t$ at $a=0.3, \varepsilon=0.4$ and $S(0)=0$ (curves $1,2,3$ correspond to $\eta(0)=0.057, \eta(0)=0.066, \eta(0)=1.0)$.

Thus, within the long-time approximation the autocorrelator tends to the stable magnitude $S_{0}$ hyperbolically, i.e.,

$$
S(t)=S_{0}+\left(\frac{2}{3}\right)(1-a) t^{-1}, \quad t \rightarrow \infty .
$$

The order parameter decreases according to the power-law dependence

$$
\eta(t)=\eta_{0}-[2(1-a)]^{2(1-a)}|m| t^{-2(1-a)}, \quad t \rightarrow \infty,
$$

where the amplitude $m$ is given by the first Eq. (32).

Thus, according to the phase portraits shown in Fig. 3(a), at $\varepsilon<\varepsilon_{0}$ the order parameter $\eta(t)$ falls down monotonically, whereas the autocorrelator can vary nonmonotonically (cf. Fig. 1). More complex behaviour appears within the domain $\varepsilon>\varepsilon_{0}$ where the ordered state occurs due to the bifurcation. As seen from Fig. 3(b), the phase plane divides into two domains corresponding to small and large values of the order parameter. Within the former, the system behaves as in the above-mentioned case $\varepsilon<\varepsilon_{0}$. If an initial magnitude of the order parameter becomes larger than critical magnitude, the system passes to the attractive point $C$. The corresponding temporal dependencies are depicted in Fig. 6. It is characteristically that these dependencies display a critical slowing-down near the separatrix $C_{0} S C$ in Fig. 3(b) (see curves 1 and 2 in Figs. 6(a), and (b)).

In order to analyse the long-time behaviour in the vicinity of the point $C$, we may not use the solution like the generalized exponent (25). The latter is applicable at non-linearity effects which are sufficient to fix the above-mentioned amplitudes $m, n$ defined by Eqs. (30), (32). In the case under consideration, the linear conditions are satisfied and instead of the generalized exponent (25) we ought to use the Mellin transformation. The principle difference is that the Mellin transformation contains a 


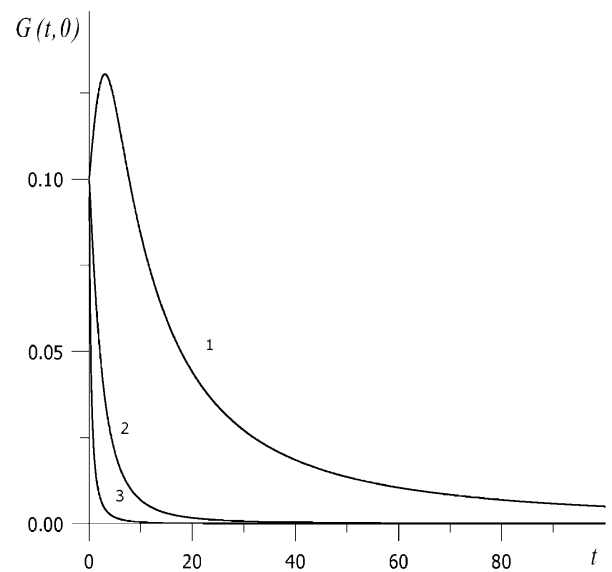

(a)

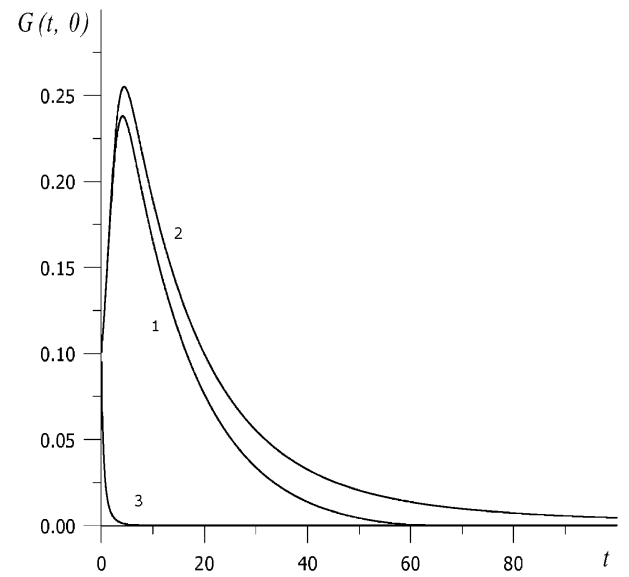

(b)

Fig. 7. Correlator $G(t, 0)$ vs. time $t$ at: (a) $a=0.3, \varepsilon=0.2, S(0)=0$ (curves 1,2,3 correspond to $\eta(0)=0.098, \eta(0)=0.4, \eta(0)=0.97$ ); (b) $a=0.3, \varepsilon=0.4, S(0)=0$ (curves 1,2,3 correspond to $\eta(0)=0.057, \eta(0)=0.066, \eta(0)=1.0)$.

set of $q$, whereas generalized exponent defines single index $q$. Inserting definitions (cf. Eqs. (28))

$$
\begin{aligned}
& \eta(t)=\eta_{0}+\int m_{q} t^{q} \mathrm{~d} q, \\
& S(t)=S_{0}+\int n_{q} t^{q} \mathrm{~d} q
\end{aligned}
$$

into linearized Eqs. (6), (20), we obtain the equations for specific amplitudes $m_{q}, n_{q} \ll 1$ :

$$
\begin{aligned}
& \left(q / t+2 \eta_{0}^{2}\right) m_{q}-3 \eta_{0} n_{q}=0, \\
& {\left[4 \eta_{0}\left(\varepsilon-\eta_{0}^{2}\right)-\alpha_{1} \eta_{0}^{p_{1}-1} p_{1}\right] m_{q}+\left[q / t+2\left(\varepsilon+\eta_{0}^{2}\right)\right] n_{q}=0 .}
\end{aligned}
$$

This system has solutions when

$$
c=\left(\varepsilon+\eta_{0}^{2}\right)\left[1 \pm \sqrt{1-\frac{8 \eta_{0}^{2}\left(2 \varepsilon-\eta_{0}^{2}\right)-3 \alpha_{1} \eta_{0}^{p_{1}} p_{1}}{\varepsilon+2 \eta_{0}^{2}}}\right],
$$

here $c=-q / t$. Thus, in a vicinity of the ordered point $C$ the dependencies of the order parameter and autocorrelator (35) and (36) take the forms

$$
\begin{aligned}
& \eta(t)=\eta_{0}+m \exp (-c t \ln t), \\
& S(t)=S_{0}+n \exp (-c t \ln t),
\end{aligned}
$$

where the amplitudes $m, n$ correspond to the index $q=-c t$.

Finally, the time dependencies of the Green function $G(t, 0)$ are determined by Eqs. (6), (7) and (20) as shown in Fig. 7. At $\varepsilon<\varepsilon_{0}$, the monotonic decrease appears for large value of initial magnitude $\eta(0)$ of the order parameter (see Fig. 7(a)). 
In the case $\varepsilon>\varepsilon_{0}$, a maximum of dependence $G(t, 0)$ disappears at condition $\eta(0)>\eta_{c}$ corresponding to the domain of the ordering state (see Fig. 7(b)).

\section{Conclusion}

Summarizing, we have derived and analysed the equations for order parameter, autocorrelator and correlation function to describe the evolution of the system with multiplicative noise. In representation of the noise amplitude as the power-law function (1), we have discussed a way to apply the cumulant expansion for average $\left\langle x^{2 a}\right\rangle$ in the case of a self-similar phase space.

We have shown that the system behaviour is given by magnitude of the exponent $a$ : at $a>\frac{1}{2}$ the system is disordered; at $a<\frac{1}{2}$ a phase transition to the ordered state is observed. In the first case the obtained time dependencies for the autocorrelator show the monotonic increasing to a stationary state, whereas the correlator shows the non-monotonic behaviour. In the second case $\left(a>\frac{1}{2}\right)$, we would investigate the system behaviour on the phase plane $(\eta, S)$. It was shown that the phase portrait divides into two domains characterized by disordered and ordered states (the former corresponds to small initial values of $\eta$, the latter - to finite values of both $\eta$ and $S$ ). We have found in what a way the system attains the steady states at long-time asymptotics. Within disordered domain, the autocorrelator decays hyperbolically and the order parameter dependence is described by the power-law function with the exponent $-2(1-a)$. In the ordered phase, the order parameter and autocorrelator exhibit exponential behaviour with index proportional to $-t \ln t$.

\section{Acknowledgements}

The authors are grateful to Charles University and Institute of Physics (Prague, Czech Republic) for the hospitality extended to them while performing this work. They are grateful to Dr. A. Khomenko for attentively reading the manuscript.

\section{References}

[1] L.D. Landau, E.M. Lifshits, Statistical Physics, Part I, Pergamon Press, Oxford, 1980.

[2] M. Turelli, Theoretical population Biology, Vol. 12, Academic, New York, 1977, p. 140.

[3] M.A. Munoz, Phys. Rev. E 57 (1998) 1377.

[4] M. Lévy, S. Solomon, Internat. J. Modern. Phys. C 7 (1996) 595.

[5] A.I. Olemskoi, in: I.M. Khalatnikov (Ed.), Physics Reviews, Part 1, Vol. 18, Gordon and Breach, Amsterdam, 1996, p. 1.

[6] A.I. Olemskoi, Physics-Uspekhi 168 (1998) 287.

[7] A.I. Olemskoi, D.O. Kharchenko, Met. Phys. Adv. Tech. 16 (1996) 841.

[8] C. Tsallis, in: S. Abe, Y. Okamoto (Eds.), Nonextensive Statistical Mechanics and its Applications, Lecture Notes in Physics, Springer, Berlin, 2000.

[9] C. Tsallis, J. Statist. Phys. 52 (1988) 479. 